\title{
Advanced tools for the safety assessment of nanomaterials
}

\author{
Bengt Fadeel', Lucian Farcal ${ }^{2}{ }^{2}$, Barry Hardy ${ }^{2}$, Socorro Vázquez-Campos ${ }^{3}$, Danail Hristozov ${ }^{4}$, \\ Antonio Marcomini ${ }^{4}$, Iseult Lynch ${ }^{5}$, Eugenia Valsami-Jones ${ }^{(15}{ }^{5}$, Harri Alenius ${ }^{1,6}$ and Kai Savolainen ${ }^{7 \star}$
}

Engineered nanomaterials (ENMs) have tremendous potential to produce beneficial technological impact in numerous sectors in society. Safety assessment is, of course, of paramount importance. However, the myriad variations of ENM properties makes the identification of specific features driving toxicity challenging. At the same time, reducing animal tests by introducing alternative and/or predictive in vitro and in silico methods has become a priority. It is important to embrace these new advances in the safety assessment of ENMs. Indeed, remarkable progress has been made in recent years with respect to mechanism-based hazard assessment of ENMs, including systems biology approaches as well as high-throughput screening platforms, and new tools are also emerging in risk assessment and risk management for humans and the environment across the whole life-cycle of nano-enabled products. Here, we highlight some of the key advances in the hazard and risk assessment of ENMs.

U ncertainty regarding the hazards and risks of ENMs for human health and the environment may hamper the exploitation and use of these novel materials. Regulatory uncertainty may also increase the cost and time needed for the development of new products. However, in a recent impact assessment report published by the European Commission (https://publications.europa. $\mathrm{eu} / \mathrm{en})$, the authors concluded that "environment, health and safety (EHS) in most sectors is considered very relevant to nanotechnology and critical to the development of the whole area," and that "the vast majority of stakeholders call for specific regulatory policies." Thus, it can be argued that nanoEHS is perceived as important and not necessarily as something that would impede innovation. However, the challenge is how to perform nano-risk assessment, and we believe that new data-intensive approaches are needed. In a landmark paper, Maynard et al. proposed five 'grand challenges' in the hope that this would galvanize research relevant to safety of nanotechnologies ${ }^{1}$. The question is whether or not the nanoEHS community has managed to address any or all of these challenges. Indeed, concerns have been raised as to the relevance of the not insignificant number of nanotoxicological studies that have been published during the past decade for risk assessment and regulation $^{2,3}$. However, we believe that the time has come for a different mindset with regards to the safety assessment of ENMs: we need to move away from the current preoccupation with insurmountable challenges and focus, instead, on the considerable progress that has been made in recent years as new and sophisticated approaches have been incorporated into nanosafety research. We need a paradigmatic shift in the way in which nanosafety assessment is conducted to cut the Gordian knot, and the purpose of this Perspective is to highlight some of the emerging tools for the hazard and risk assessment of ENMs.

A specific challenge in risk assessment of ENMs pertains to the methods used for the assessment of ENM-associated hazards and risks, as ENMs differ markedly from traditional chemicals: while ENMs are chemicals, they are also more than chemicals, and their physicochemical properties may vary considerably ${ }^{4,5}$. Indeed, one of the key lessons from the first decade of nanosafety research is that both the synthetic (material-intrinsic) and the acquired or contextdependent 'identity' of ENMs should be carefully characterized ${ }^{6,7}$. Furthermore, while there have been significant global investments in nanosafety research that have led to a better understanding of ENM-induced hazards and the underlying mechanisms, it continues to be difficult to come to well-justified conclusions regarding the risks of ENMs in the current regulatory setting. The fact that the member states have now (April 2018) agreed to make provisions for ENMs in the European chemicals legislation, REACH, is highly significative. These amendments will address the current knowledge gap regarding which ENMs are placed on the market and in which quantities. This is an important step forward. However, we believe that hazard assessment of ENMs can be markedly improved by implementing new approaches with which to ascertain or predict the relationship between ENM properties and toxicity ${ }^{8,9}$ and this, in turn, may inform grouping and read-across of ENMs.

There are ongoing attempts (Box 1 ) to provide a reliable foundation for the prediction of ENM hazards, though it is obvious that current attempts require further refinements to fulfil the regulatory requirements, based in many cases on quantitative hazard and risk assessment of ENMs. The major challenges in promoting quantitative assessment of hazards and risks of ENMs are the laborious process of generating data on the dose-response of ENMs for multiple toxicity end-points and the difficulties in correlating these results to actual exposure levels in various settings, including workplaces, consumer exposure and in the environment ${ }^{10-12}$. Furthermore, there have been large coordinated efforts to harmonize current methods for ENM hazard and risk assessment. One of the key undertakings in this area has been the NANoREG project with 48 partner institutes and a budget of 50 MEuro (www.nanoreg.eu). The project aimed to address uncertainties related to how one should judge the EHS aspects of ENMs in a regulatory context. It thus focused on identifying what nano-specific EHS aspects are relevant from

IInstitute of Environmental Medicine, Karolinska Institutet, Stockholm, Sweden. ${ }^{2}$ Douglas Connect GmbH, Basel, Switzerland. ${ }^{3}$ Department of New Technologies, LEITAT, Barcelona, Spain. ${ }^{4}$ Department of Biology, University of Venice Ca Foscari, Venice, Italy. ${ }^{5}$ School of Geography, Earth and Environmental Sciences, University of Birmingham, Birmingham, UK. ${ }^{6}$ Department of Bacteriology and Immunology, University of Helsinki, Helsinki, Finland. ${ }^{7}$ Finnish Institute of Occupational Health, Helsinki, Finland. *e-mail: Kai.Savolainen@ttl.fi 


\section{Box 1 Cooperation in nanosafety research in the EU and beyond}

The EU Nanosafety Cluster (www.nanosafetycluster.eu) is a forum for EU-funded projects addressing the safety of nanomaterials and nanotechnologies. The main aims are to promote synergy among these projects and foster collaboration for maximizing impact, policy elaboration and international cooperation. The forum is an initiative of the European Commission DirectorateGeneral for Research and Innovation. The Nanosafety Cluster Compendium, updated on an annual basis and available on the website, provides a concise snapshot of all the current and recent projects. Two large projects, FP7-NanoMILE (www.nanomile. eu-vri.eu) and FP7-NANOSOLUTIONS (www.nanosolutionsfp7.com) focused on new tools for hazard assessment of ENMs including HTS and systems biology-based approaches, while FP7-eNanoMAPPER (www.enanomapper.net) aimed at creating a community framework and a common language to accelerate cooperation. Several new projects are underway in the Horizon 2020 programme, including NanoFASE (www.nanofase.eu), a project that aims to develop an exposure assessment framework to evaluate the environmental fate of nanomaterials, and caLIBRAte (www.nanocalibrate.eu), a consortium that seeks to develop a risk governance framework for ENM and nano-enabled products. The newly launched European Union Observatory for Nanomaterials (www.euon.echa.europa.eu) hosted by the European Chemicals Agency, provides a useful repository of information related to safety, innovation and use of ENM. The US-EU nanoEHS dialogue or platform (www.us-eu.org), in its turn, has as its main goals to promote an active discussion about environmental health and safety (EHS) questions for nano-enabled products, encourage joint programs that would leverage resources, and support the communities of research-largely selfmanaged working groups for scientists and other stakeholders.

a regulatory point of view, on carrying out the research needed to fill in the gaps, and on developing a framework and a 'toolbox' for hazard and risk assessment of ENMs. Other EU-funded projects (Box 1) have employed emerging methods including high-throughput screening and systems biology/toxicology approaches to develop a mechanistic understanding of ENM hazards for human health and the environment. Taken together, these efforts are likely to enable a major leap forward, as compared with the current material-bymaterial approach, and will provide the basis for a more general feature-driven hazard assessment of ENMs relying on a palette of methods that provide information on ENM physicochemical properties, toxicity features and alterations of gene and protein expression, and on finding associations between relevant data layers by applying bioinformatics tools. These novel approaches have not yet received regulatory acceptance, but they may pave the way for quicker, and more affordable and reliable risk assessment of ENMs.

\section{Harmonized ontologies and data-sharing practices}

It has become increasingly clear that the generation, representation, evaluation and communication of safety-related evidence on chemicals, including ENMs, requires the establishment of a knowledge infrastructure supported by harmonized methods, ontologies (languages) and data science practices ${ }^{13}$. Nanotechnology, in particular, poses significant challenges due to the complexity of the materials and the characterization of their interactions with biological systems. Therefore, an infrastructure for transparent data sharing and data analysis, and the creation of computational toxicology models for ENMs has been proposed within the EU NanoSafety Cluster (of nanosafety projects) (Box 1) to support the nanosafety community. These efforts are also being aligned with other international research efforts including those in the United States, through the so-called US-EU nanoEHS platform (Box 1).
Indeed, the need for a federated database system linking and transparently integrating all available information sources related to nanosafety is illustrated by the recently established European Union Observatory for Nanomaterials, hosted by the European Chemicals Agency. This umbrella resource (Box 1) aims to provide an entry point to the respective repositories of EU-funded research results, models and tools, and bring together the community knowledge, thereby reducing its fragmentation. Furthermore, to pursue the optimal usage of this data, a system should consider the FAIR data principles of findability, accessibility, interoperability and reusability of data and the algorithms, tools and workflows that operate on it. Therefore, recent community efforts have focused on building such computational frameworks and infrastructures for ENMs, adopting an interoperable design and enabling effective and integrated approaches in risk assessment. Researchers at the Joint Research Centre of the European Commission recently published a state-ofthe-art review of the currently available computational approaches for safety assessment of ENMs ${ }^{14}$.

Another major challenge for the nanosafety community is the establishment of common languages, standards and harmonized infrastructures relevant to the needs of stakeholders in the nanosafety area. Ontologies, that is, structured, controlled vocabularies, are needed to support the automation of information systems and link these to the chemical and biological properties of ENMs. The EU-funded project, eNanoMapper, and other international initiatives have worked towards developing nano-specific ontologies allowing the standardization of the terminology in the nanosafety domain, and developed a substantial vocabulary to be used in nanotechnology and safety assessment ${ }^{15}$. This ontology can be used for harmonization purposes. To exemplify, the infrastructure developed by the eNanoMapper project is supported by such agreed (nano-specific) language, on an open platform for integrating and accessing different ENM data sources ${ }^{16}$. Several other EU projects have adhered to the same approach, including NANoREG, and the aim is to make the database widely available to the community (Fig. 1). Data from other, large projects such as MARINA have been merged into this database and could provide a useful resource for predictive modelling ${ }^{17}$. Furthermore, several Horizon 2020 projects, including caLIBRAte (Box 1), have now adopted the eNanoMapper approach for data collection, data curation and data sharing. The effective and reliable exchange of information among different projects, in the EU and beyond, and between data providers and data users requires the establishment of minimum criteria for data content and for data quality that is supported by the ontology and controlled vocabularies, which need to be sustained in the upcoming years. An important role in advancing this area is played by the US-EU nanoEHS platform (Box 1). This transatlantic forum has facilitated the nanosafety harmonization process leading to a consensus on approaches for data management and ontologies. As a consequence, there is consensus between different international initiatives with regards to the data structure, and a good alignment of computing standards. These efforts will enable a sustainable and harmonized knowledge infrastructure supporting the risk assessment of ENMs and nano-enabled products.

\section{High-throughput screening to speed up hazard assessment} Nanosafety research has largely proceeded through a material-bymaterial approach using conventional, low-throughput assays established to assess well-known toxicity end-points including reactive oxygen species generation, cytotoxicity (cell death/cell viability) and DNA damage. However, there is a need to develop assays that reflect more closely the mode-of-action of ENMs, and to develop assays for rapid screening of large numbers of ENMs at different concentrations and under different exposure conditions. The deployment of such high-content assays (HCA) and high-throughput screening (HTS) approaches could yield substantial savings in terms of time 


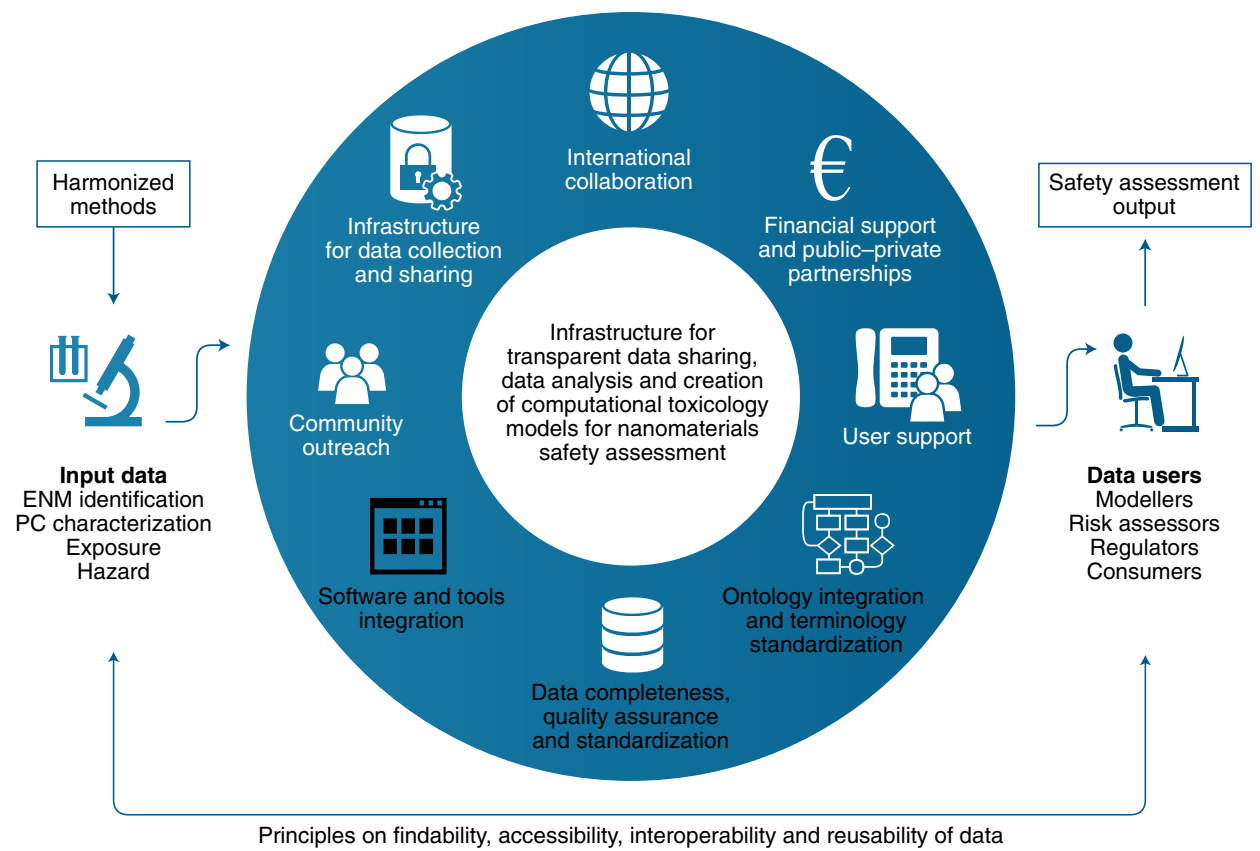

Fig. 1 | Enabling a harmonized knowledge infrastructure supporting environment, health and safety (EHS) assessment of nanomaterials. ENM, engineered nanomaterial; PC, physicochemical.

and money ${ }^{18}$. HTS is well established in drug discovery, though it is noted that regulatory decisions are not made on the basis of such data as yet. Additionally, HTS is increasingly being applied to support predictive testing of chemicals, as evidenced by the ToxCast programme of the US Environmental Protection Agency ${ }^{19}$. This aligns well with current efforts to promote mechanism-based in vitro assays and in silico predictive tools as elements of an alternative testing strategy for chemicals and newly emerging substances including $\mathrm{ENMs}^{20}$. Capitalizing on HCA/HTS approaches, datasets are generated that have the potential to enable ranking of ENM hazards and may allow for the development of predictive models of nanotoxicity ${ }^{21-24}$. Automated screening of zebra fish embryos was reported as a feasible approach to anchor in vitro screening results of $\mathrm{ENMs}^{25,26}$.

It is worth noting that HTS can be applied in a tiered approach to evaluate a few key end-points (for example, cytotoxicity) to study dose-response relationships for a large number of ENMs, followed by further characterization of relevant effects such as genotoxicity and immunotoxicity using more advanced methods. Alternatively, the most important hazard-associated features are first identified using omics approaches and advanced computational methods. Then, based on these minimal but most informative sets of hazardassociated features, a platform for HTS can be developed enabling rapid, accurate and affordable generation of data necessary for ENM hazard prediction (Fig. 2). Both approaches were explored in the recent EU-funded projects, NanoMILE and NANOSOLUTIONS (Box 1). The major requirement for all computational approaches is the need for high-quality data that is as complete as possible in terms of physicochemical characterization of the ENM, both in the pristine form and under the relevant exposure conditions. In addition, the potential for interference of ENMs with the toxicity assays needs to be considered ${ }^{27}$. Key elements of HTS approaches for ENM testing include the establishment of reference ENM libraries with systematically varying physicochemical properties, enabling a mechanistic understanding of the drivers of ENM toxicity ${ }^{28}$. A major challenge is that varying one ENM property often requires changes to the synthesis method and this may inadvertently result in other physicochemical properties changing also. Thus, tailored libraries have been developed where the goal has been to ensure that all but one physicochemical property remains constant. One such library, developed in the frame of the NanoMILE project, included a series of metal oxide cores of different compositions, different core sizes and different capping ligand sizes; the combination of properties yielded nine variants ${ }^{29}$. Notably, artificially aged ENMs were almost invariably less toxic than the pristine materials. Nevertheless, HTS is a powerful approach for screening of the hazard potential of large numbers of pristine ENMs, provided that robust assays are used and meaningful (validated) end-points are tested. Indeed, HCA and/or HTS approaches can be utilized to probe the mechanism of action of ENM via targeted assays ${ }^{30}$.

In the NANOSOLUTIONS project, a panel of more than 30 ENMs was generated with variations in core chemistry and surface functionalization, and these materials were subjected both to conventional toxicity testing and HTS based on six different end-points, using the same cell model ${ }^{31,32}$. The latter exercise demonstrated a good concordance between conventional cyto- and genotoxicity end-points and HTS, though the latter approach was far more rapid. In other related work, a library of 105 surface-modified gold nanoparticles was generated ${ }^{33}$ to study the role of the biocorona in cellular uptake, though the approach was not high-throughput. Nevertheless, the latter study points towards a predictive testing paradigm as opposed to a case-by-case approach.

\section{Emerging systems biology approaches in nanosafety}

Classical toxicological testing paradigms still rely heavily on animal testing, despite societal pressures to switch to alternative in vitro test methods. The twenty-first century toxicology paradigm ${ }^{34}$ calls for a shift away from descriptive toxicology, based to a large extent on animal testing of toxicants one-by-one and with a multitude of functionally disconnected assays, towards a predictive toxicology grounded in a more solid understanding of the relevant toxicity and modes of action in humans, or the environment. Systems biology can aid in this endeavour. Indeed, systems toxicology approaches combining advanced analytical and computational tools can provide quantitative information on systems-wide molecular changes in the context of toxicant exposure, leading to information on how 


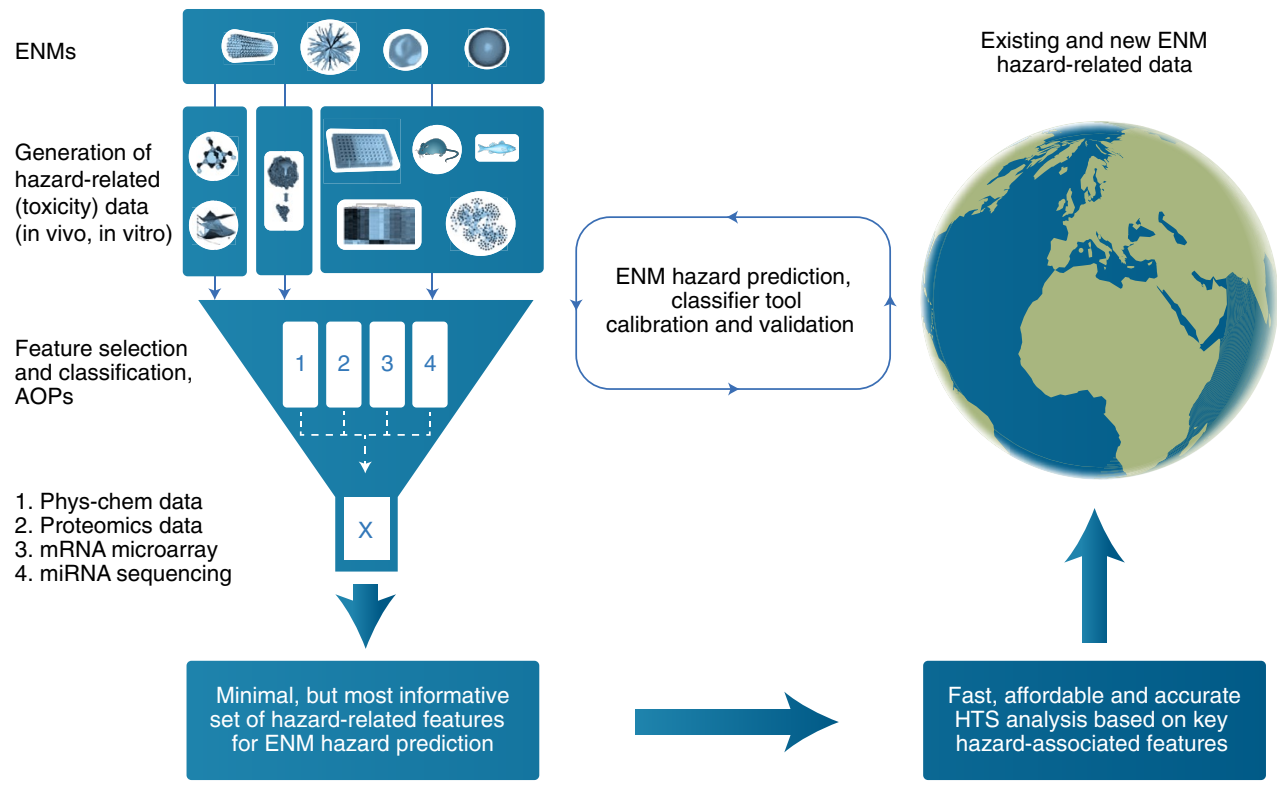

Fig. 2 | Systems biology paradigm for ENM hazard and risk assessment focusing on the identification of a minimal yet most informative set of features with which to predict toxicity. AOP, adverse outcome pathway; ENM, engineered nanomaterial; HTS, high-throughput screening.

biological networks are perturbed by toxicants ${ }^{35}$. Systems toxicology aims to change the way in which adverse effects of chemicals or other toxicants are characterized, from isolated empirical end-points to integrated pathways of toxicity ${ }^{36}$. In the context of nanosafety, systems toxicology promises to shed new light on the interactions of ENMs with biological systems, and reveal the causal connection between changes in the expression of genes, proteins or metabolites and the biological pathways that underlie the toxicity phenotypes ${ }^{37}$. However, due to relatively high cost, requirement of highly specialized equipment, and slow analysis speed per sample, only a few biological replicates are usually analysed. Global profiling of gene or protein expression and subsequent computational analysis of the data provides unprecedented insight into toxicological mechanisms, but the approach cannot be viewed as high-throughput. Moreover, it is challenging to separate ENM-induced biologically relevant perturbations from artificially induced small variations unless research design, analysis strategy and quality issues are strictly controlled. Key challenges are also how to link reliably identified gene profiles/ networks to toxicological phenotypes (for example, immunotoxicity, genotoxicity and so on), and how to demonstrate reliability and prediction accuracy of computational models in real life. The objective is not to generate lists of genes or proteins that are up- or downregulated, but to develop predictive models. In an illustrative, recent study, in silico-based knowledge extraction and modelling was applied to develop a 'data fusion pipeline' comprising diverse types of data including omics results to construct novel adverse outcome pathway descriptions for pulmonary fibrosis ${ }^{38}$. Adverse outcome pathways are representations of toxicological data that can be used to support risk assessment.

Huge amounts of information are generated in omics experiments. To identify the hazard-relevant molecular features (signatures), one needs to be able to isolate the relevant information while taking into account the statistical dependency between the variables. In such a context, the group of features that best predicts the safety of ENMs might not be composed of elements derived from only one data layer, such as the intrinsic physicochemical properties of ENMs, but also by features with a combinatorial effect derived from multiple layers of data. Thus, by systematically integrating multiple layers of experimental data together with information extrapolated from the relevant literature, a more robust group- ing of ENMs and hazard prediction can be achieved (Fig. 2). The NANOSOLUTIONS project aimed to generate a computer algorithm capable of predicting the safety of ENMs based on a minimal but informative set of features selected across multiple data layers. Based on integration of data from several different omics layers (that is, transcriptomics, proteomics, epigenomics), physicochemical properties and proteomics-based biocorona profiling, along with in vitro and in vivo toxicity data for a panel of more than 30 ENMs, a classifier algorithm designated the ENM safety classifier, composed only of approximately one dozen most informative hazard associated features, was generated that is capable of predicting ENM toxicity with high accuracy ${ }^{39}$. This computational predictive approach represents a major step forward and may enable hazard classification based on a relatively small number of toxicity tests of ENMs. In another study, the consortium developed a computational tool termed INSIdE nano ('integrated network of systems biology effects of nanomaterials') for contextualizing ENM modes-ofaction with respect to human diseases, drug treatments and chemical exposures, based on the integration of extensive genome-wide transcriptomics data. This novel computational tool allows for the inference or prediction of connections between ENMs and diseases on the basis of transcriptomics-based signatures (A. Serra et al., submitted manuscript)

Omics-based approaches can be used not only to support hazard identification and classification of ENMs, but can also be used to develop biomarkers of exposure or effect. In ref. ${ }^{40}$, researchers exposed a human cell line versus mouse lungs to several carbonbased nanomaterials and found that the effects, when investigated at the level of significantly altered molecular functions, displayed considerable similarities in the transcriptomic responses. In another recent study, genome-wide usage of gene transcription start sites and linked active enhancer regions was analysed in the lungs of mice exposed to multi-walled carbon nanotubes (MWCNTs), and a detailed picture of the biological effects of MWCNT exposure emerged, suggesting the identification of biomarkers for early detection of MWCNT-induced inflammation ${ }^{41}$. One common theme emerging from this selective sampling of the literature is that the bioinformatics approaches applied are of critical importance, and that experimental and computational methods must be developed in tandem. Common practices for data collection and good 


\section{Box 2 | New web-based tools for risk assessment of nanomaterials}

The EU-funded projects, FP7-SUN (www.sun-fp7.eu) and FP7-GUIDEnano (www.guidenano.eu) developed two complementary web-based risk assessment tools for ENM: the GUIDEnano Tool and the SUN Decision Support System (SUNDS). The SUNDS software system can estimate occupational, consumer and environmental risks from ENM in industrial products along their life-cycle. In situations when the risks are not acceptable, SUNDS proposes suitable risk management measures, including information about their costs compared to the benefits of the nanotechnologies. Risk control can be demonstrated by reducing risk to below threshold levels or by investigating feasible alternatives to the substance. If risks are not adequately controlled and no feasible alternatives to a substance are found, socioeconomic analysis can be performed to demonstrate that benefits of using a certain ENM significantly outweigh the costs. The GUIDEnano Tool guides the nano-enabled product developers with regard to the design and application of the most appropriate risk assessment and mitigation strategy for a specific product. The correct implementation of this guidance ensures that the risks associated with a nano-enabled product, throughout its whole life-cycle, have been appropriately evaluated and mitigated to an acceptable level, according to the most recent knowledge at the time of implementation. The GUIDEnano Tool combines a range of predictive models, multilevel decision trees and databases to derive critical information along the risk assessment process. The modular structure of the tool allows the user to define the scope of each risk assessment, which can range from a specific exposure scenario to the entire product life-cycle, considering both human and environmental risks. The GUIDEnano Tool is undergoing further performance testing using industrial case studies in the caLIBRAte project, and SUNDS will be adapted for biomaterials used for medical applications in the newly launched Horizon 2020 project, BIORIMA (www.biorima.eu).

alignment of computational approaches are needed (discussed above). Other key challenges in the future are to develop easy-touse advanced hazard prediction tools that are predictive not only in a short-term, high-dose exposure scenario, but also in predicting hazardous effects in the long term arising from more realistic, lowdose exposures.

\section{New web-based tools for risk-benefit assessment}

Quantitative risk assessment and setting of safety limit values for ENMs has not yet been possible due to data gaps ${ }^{42}$. Similarly, attempts to carry out reliable evaluations on possible harmful effects of ENMs at different stages of the product life-cycle have been hampered by a lack of data. In general, existing methodologies and tools focus mainly on exposure assessment in the production and manufacturing stages of the life-cycle, neglecting exposure that occurs during the use and end-of-life stages. Recent efforts by two European research projects (SUN and GUIDEnano) (Box 2) to integrate the existing state-of-the-art knowledge/data has resulted in the development of two different, yet complementary webbased risk assessment tools: the GUIDEnano Tool ${ }^{43}$ and the SUN Decision Support System (SUNDS) ${ }^{44,45}$. Implementation of these tools may provide a step forward in the evaluation and mitigation of risks associated with nano-enabled products throughout their whole life-cycle ${ }^{46}$. The flexibility of these tools allows the user to define the scope of each risk assessment, ranging from a specific exposure scenario to the entire product life-cycle, considering both human and environmental risks (Box 2). This information flows through the different modules of the tools to provide a quantitative risk assessment for each scenario of interest. Depending on the outcome of the risk assessment, a range of risk mitigation measures can then be considered. The SUN tool can also assess the benefits of nano-enabled products by means of a structured methodology for socio-economic analysis, as anticipated, for instance, under $\mathrm{REACH}$. These options are likely to be of significant practical value particularly for small and medium-sized enterprises because they would enable the integration of technical data about risks, benefits and costs of nano-enabled products. Hence, these web-based tools would allow the construction of sustainability portfolios to support informed decisions about safer production, handling and end-of-life treatment of ENMs, and whether or not to invest in the production of specific nano-enabled products. The successful exploitation of these tools obviously relies on the availability of high-quality physicochemical, exposure and hazard data for ENMs. The cost-effective acquisition of such data can be facilitated by HTS supported by grouping and read-across approaches ${ }^{47}$. The implementation of grouping and read-across could enhance the efficiency of testing for risk assessment and could also promote and inform safer design of nano-enabled products. Furthermore, while the path may be long and winding, the translation of biomarkers derived from omics studies into routine assays useful in risk assessment would be a major step towards affordable and quicker risk assessment of ENMs. Overall, we recommend a shift from the current risk control to a risk prevention-based paradigm. This can be achieved by focusing the available resources on designing out undesired material and/ or process characteristics in early stages of product development.

It is critically important to assess and quantify the global environmental impact of nano-enabled products using life-cycle analysis approaches ${ }^{48}$. Identification and quantification of released materials at each stage of the nano-enabled product life cycle, from cradle to grave, has been an important focus of the nanosafety research agenda in recent years ${ }^{49-51}$. The generation of knowledge and data on these released materials, their transport and transformations in different human and environmental compartments, as well as their potential toxic impact, constitutes a common goal for risk assessment and life-cycle assessment of ENMs. The recent projects, SUN and GUIDEnano (Box 2) have contributed towards these efforts by working on case studies comprising different nano-enabled products taking into consideration the different stages of their life cycle $^{52}$. Some authors have advocated that risk assessment and life-cycle analysis should be merged at the methodological level ${ }^{53}$, while others have argued that it is not meaningful to merge the two approaches as they are suited for different purposes and address different questions ${ }^{54}$. In a recent Perspective in this journal ${ }^{55}$, the strengths and weaknesses of each of these methods as well as the arguments for and against combining the two approaches were reviewed and it was concluded that more collaboration is needed. In SUNDS, risk assessment and socioeconomic analysis is integrated to establish the risk-benefit balance of nano-enabled products throughout their life-cycle (Box 2).

\section{Conclusions}

During the past several years, remarkable progress has been made in understanding the mechanisms underlying the biological effects of ENMs. Material characterization of ENMs has, in general, been much improved and is now routinely documented in scientific publications and this has enabled the identification of associations between ENM physicochemical properties and perturbations of biological systems. However, further testing of ENMs that have undergone aging or transformation through the life-cycle of nanoenabled products is needed ${ }^{56}$.

The Organisation for Economic Co-operation and Development working party on manufactured nanomaterials recently concluded that the approaches for testing of traditional chemicals are, overall, appropriate for assessing the safety of ENMs, but it was also 
noted that some test guidelines need to be adapted or amended for $\mathrm{ENMs}^{57}$. In our opinion, adaptation of traditional assays is not sufficient; we also need to speed up testing using validated in vitro assays based on relevant end-points, that is, in vitro end-points that adequately mirror in vivo outcomes. To this end, emerging technologies, especially omics and high-throughput and/or high-content screening platforms, coupled with bioinformatics or computational approaches, have enabled the analysis of large amounts of data and the identification of meaningful associations from the endless possible permutations existing in the data collections. In this context, it is worth noting that omics-based systems biology approaches are not 'hypothesis-free', but instead serve to generate hypotheses or predictions for further validation ${ }^{58}$. This is, in essence, the purpose of modelling. In addition, a more complete understanding of associations between ENM characteristics and biological features may enable safe-by-design approaches that would greatly improve ENM safety and most likely also foster public trust toward these materials and the technologies utilizing them. Furthermore, structured approaches for the evaluation and mitigation of risks associated with nano-enabled products throughout their life-cycle are also being developed. Together, these efforts could enable reliable, science-driven, quantitative assessment of risks of ENMs and result in the identification of a minimum set of information required for predicting hazards and risks for health and the environment induced by exposure to ENMs. This, in turn, would greatly promote the safe and sustainable use of ENMs and effectively boost ENMdriven innovations.

Received: 20 November 2017; Accepted: 5 June 2018; Published online: 6 July 2018

\section{References}

1. Maynard, A. D. et al. Safe handling of nanotechnology. Nature 444, 267-269 (2006).

2. Krug, H. F. Nanosafety research-are we on the right track? Angew. Chem. Int. Ed. Engl. 53, 12304-12319 (2014).

3. Valsami-Jones, E. \& Lynch, I. NANOSAFETY. How safe are nanomaterials? Science 350, 388-389 (2015).

4. Sayes, C. M. \& Warheit, D. B. Characterization of nanomaterials for toxicity assessment. Wiley Interdiscip. Rev. Nanomed. Nanobiotechnol. 1, 660-670 (2009).

5. Fadeel, B., Fornara, A., Toprak, M. S. \& Bhattacharya, K. Keeping it real: the importance of material characterization in nanotoxicology. Biochem. Biophys. Res. Commun. 468, 498-503 (2015).

6. Walkey, C. D. \& Chan, W. C. Understanding and controlling the interaction of nanomaterials with proteins in a physiological environment. Chem. Soc. Rev. 41, 2780-2799 (2012)

7. Monopoli, M. P., Åberg, C., Salvati, A. \& Dawson, K. A. Biomolecular coronas provide the biological identity of nanosized materials. Nat. Nanotech. 7, 779-786 (2012).

8. Cohen, Y., Rallo, R., Liu, R. \& Liu, H. H. In silico analysis of nanomaterials hazard and risk. Acc. Chem. Res. 46, 802-812 (2013).

9. Winkler, D. A. Recent advances, and unresolved issues, in the application of computational modelling to the prediction of the biological effects of nanomaterials. Toxicol. Appl. Pharmacol. 299, 96-100 (2016).

10. Fadeel, B. et al. There's plenty of room at the forum: potential risks and safety assessment of engineered nanomaterials. Nanotoxicology 1, 73-84 (2007).

11. Savolainen, K. et al. Risk assessment of engineered nanomaterials and nanotechnologies-a review. Toxicology 269, 92-104 (2010).

12. Hussain, S. M. et al. At the crossroads of nanotoxicology in vitro: past achievements and current challenges. Toxicol. Sci. 147, 5-16 (2015).

13. Valsami-Jones, E., Lynch, I. \& Charitidis, C. A. Nanomaterial ontologies for nanosafety: a rose by any other name.... J. Nanomed. Res. 3, 00070 (2016).

14. Worth, A. et al. Evaluation of the Availability and Applicability of Computational Approaches in the Safety Assessment of Nanomaterials (Joint Research Centre, 2017); https://doi.org/10.2760/248139

15. Hastings, J. et al. eNanoMapper: harnessing ontologies to enable data integration for nanomaterial risk assessment. J. Biomed. Semant. 6, 1-15 (2015).

16. Jeliazkova, N. et al. The eNanoMapper database for nanomaterial safety information. Beilstein J. Nanotechnol. 6, 1609-1634 (2015).

17. Farcal, L. et al. Comprehensive in vitro toxicity testing of a panel of representative oxide nanomaterials: first steps towards an intelligent testing strategy. PLoS ONE 10, e0127174 (2015).
18. Collins, A. R. et al. High throughput toxicity screening and intracellular detection of nanomaterials. Wiley Interdiscip. Rev. Nanomed. Nanobiotechnol. 9, e1413 (2017).

19. Richard, A. M. et al. ToxCast chemical landscape: paving the road to 21 st century toxicology. Chem. Res. Toxicol. 29, 1225-1251 (2016).

20. Nel, A. E. \& Malloy, T. F. Policy reforms to update chemical safety testing. Science 355, 1016-1018 (2017)

21. Anguissola, S., Garry, D., Salvati, A., O’Brien, P. J. \& Dawson, K. A. High content analysis provides mechanistic insights on the pathways of toxicity induced by amine-modified polystyrene nanoparticles. PLOS ONE 9, e108025 (2014)

22. Harris, G. et al. Iron oxide nanoparticle toxicity testing using highthroughput analysis and high-content imaging. Nanotoxicology $\mathbf{9}$, 87-94 (2015).

23. Liu, R. et al. Classification NanoSAR development for cytotoxicity of metal oxide nanoparticles. Small 7, 1118-1126 (2011).

24. Rallo, R. et al. Self-organizing map analysis of toxicity-related cell signaling pathways for metal and metal oxide nanoparticles. Environ. Sci. Technol. 45, 1695-1702 (2011)

25. George, S. et al. Use of a high-throughput screening approach coupled with in vivo zebrafish embryo screening to develop hazard ranking for engineered nanomaterials. ACS Nano 5, 1805-1817 (2011).

26. Liu, R. et al. Automated phenotype recognition for zebrafish embryo based in vivo high throughput toxicity screening of engineered nano-materials. PLoS ONE 7, e35014 (2012).

27. Marchese Robinson, R. L. et al. How should the completeness and quality of curated nanomaterial data be evaluated? Nanoscale 8, 9919-9943 (2016).

28. Nel, A. et al. Nanomaterial toxicity testing in the 21st century: use of a predictive toxicological approach and high-throughput screening. Acc. Chem. Res. 46, 607-621 (2013).

29. Briffa, S. M. et al. Development of scalable and versatile nanomaterial libraries for nanosafety studies: polyvinylpyrrolidone (PVP) capped metal oxide nanoparticles. RSC Adv. 7, 3894-3906 (2017).

30. Hansjosten, I. et al. Microscopy-based high-throughput assays enable multi-parametric analysis to assess adverse effects of nanomaterials in various cell lines. Arch. Toxicol. 92, 633-649 (2018).

31. Gallud, A. et al. Cytotoxicity screening of a panel of 31 nanomaterials in the human monocytic cell line THP.1 versus primary human monocyte-derived macrophages: assessing the role of surface modification. In New Tools and Approaches for Nanomaterial Safety Assessment: Book of Abstracts (2017); https://doi.org/10.13140/RG.2.2.26504.62722

32. Hongisto, V. et al. High-throughput screening approach evaluated toxicity of 31 engineered nanomaterials generated for the NANOSOLUTIONS project. In New Tools and Approaches for Nanomaterial Safety Assessment: Book of Abstracts (2017); https://doi.org/10.13140/RG.2.2.26504.62722

33. Walkey, C. D. et al. Protein corona fingerprinting predicts the cellular interaction of gold and silver nanoparticles. ACS Nano $\mathbf{8}$, 2439-2455 (2014).

34. Collins, F. S., Gray, G. M. \& Bucher, J. R. TOXICOLOGY. Transforming environmental health protection. Science 319, 906-907 (2008).

35. Sturla, S. J. et al. Systems toxicology: from basic research to risk assessment. Chem. Res. Toxicol. 27, 314-329 (2014).

36. Hartung, T. et al. Systems toxicology: real world applications and opportunities. Chem. Res. Toxicol. 30, 870-882 (2017).

37. Costa, P. M. \& Fadeel, B. Emerging systems biology approaches in nanotoxicology: towards a mechanism-based understanding of nanomaterial hazard and risk. Toxicol. Appl. Pharmacol. 299, 101-111 (2016).

38. Nymark, P. et al. A data fusion pipeline for generating and enriching adverse outcome pathway descriptions. Toxicol. Sci. 162, 264-275 (2018).

39. Fortino, V. \& Greco, D. ENM SAFETY CLASSIFIER - a multi-view feature selection and classification algorithm for prediction of engineered nanomaterials (ENM) safety. In New Tools and Approaches for Nanomaterial Safety Assessment: Book of Abstracts (2017); https://doi.org/10.13140/ RG.2.2.26504.62722

40. Kinaret, P. et al. Network analysis reveals similar transcriptomic responses to intrinsic properties of carbon nanomaterials in vitro and in vivo. ACS Nano 11, 3786-3796 (2017).

41. Bornholdt, J. et al. Identification of gene transcription start sites and enhancers responding to pulmonary carbon nanotube exposure in vivo. ACS Nano 11, 3597-3613 (2017).

42. Hristozov, D. R., Gottardo, S., Critto, A. \& Marcomini, A. Risk assessment of engineered nanomaterials: a review of available data and approaches from a regulatory perspective. Nanotoxicology 6, 880-898 (2012).

43. Park, M. et al. Hazard evaluation in GUIDENANO: a web-based guidance tool for risk assessment and mitigation of nano-enabled products. In New Tools and Approaches for Nanomaterial Safety Assessment: Book of Abstracts (2017); https://doi.org/10.13140/RG.2.2.26504.62722

44. Subramanian, V. et al. Sustainable nanotechnology decision support system: bridging risk management, sustainable innovation and risk governance. J. Nanopart. Res. 8, 1-13 (2016) 
45. Zabeo, A. et al. SUNDS, a multi-criteria decision support system for nanotechnology risk assessment and management. In New Tools and Approaches for Nanomaterial Safety Assessment: Book of Abstracts (2017); https://doi.org/10.13140/RG.2.2.26504.62722

46. Dekkers, S. et al. Towards a nanospecific approach for risk assessment. Regul. Toxicol. Pharmacol. 80, 46-59 (2016).

47. Oomen, A. G. et al. Grouping and read-across approaches for risk assessment of nanomaterials. Int. J. Environ. Res. Public Health 12 13415-13434 (2015)

48. González-Gálvez, D., Janer, G., Vilar, G., Vílchez, A. \& Vázquez-Campos, S. The life cycle of engineered nanoparticles. Adv. Exp. Med. Biol. 947, 41-69 (2017).

49. Fernández-Rosas, E. et al. Influence of nanomaterial compatibilization strategies on polyamide nanocomposites properties and nanomaterial release during the use phase. Environ. Sci. Technol. 50, 2584-2594 (2016).

50. Mitrano, D. M., Lombi, E., Dasilva, Y. A. \& Nowack, B. Unraveling the complexity in the aging of nanoenhanced textiles: a comprehensive sequential study on the effects of sunlight and washing on silver nanoparticles. Environ. Sci. Technol. 50, 5790-5799 (2016).

51. Wohlleben, W. et al. A pilot interlaboratory comparison of protocols that simulate aging of nanocomposites and detect released fragments. Environ. Chem. 11, 402-418 (2014).

52. Nowack, B. et al. Meeting the needs for released nanomaterials required for further testing - the SUN approach. Environ. Sci. Technol. 50, 2747-2753 (2016).

53. Tsang, M. P., Kikuchi-Uehara, E., Sonnemann, G. W., Aymonier, C. \& Hirao, M. Evaluating nanotechnology opportunities and risks through integration of life-cycle and risk assessment. Nat. Nanotech. 12, 734-739 (2017).

54. Linkov, I. et al. Integrate life-cycle assessment and risk analysis results, not methods. Nat. Nanotech. 12, 740-743 (2017).
55. Guinée, J. B., Heijungs, R., Vijver, M. G. \& Peijnenburg, W. J. G. M. Setting the stage for debating the roles of risk assessment and life-cycle assessment of engineered nanomaterials. Nat. Nanotech. 12, 727-733 (2017).

56. Bishop, L. et al. In vivo toxicity assessment of occupational components of the carbon nanotube life cycle to provide context to potential health effects. ACS Nano 11, 8849-8863 (2017).

57. Rasmussen, K. et al. Review of achievements of the OECD Working Party on Manufactured Nanomaterials' Testing and Assessment Programme. From exploratory testing to test guidelines. Regul. Toxicol. Pharmacol. 74, 147-160 (2016).

58. Fadeel, B. Systems biology in nanosafety research. Nanomed. (Lond.) 10, 1039-1041 (2015)

\section{Acknowledgements}

The authors and their laboratories were supported, in part, by the European Commission through the Seventh Framework Programme (FP7-eNANOMAPPER, grant no. 604134; FP7-GUIDENANO, grant no. 604387; FP7-NANOMILE, grant no. 310451; FP7NANOSOLUTIONS, grant no. 309329; FP7-SUN, grant no. 604305). We thank all the project partners for invaluable contributions to these projects, and the project officers for their guidance.

\section{Competing interests}

The authors declare no competing interests.

\section{Additional information}

Reprints and permissions information is available at www.nature.com/reprints.

\section{Correspondence should be addressed to K.S.}

Publisher's note: Springer Nature remains neutral with regard to jurisdictional claims in published maps and institutional affiliations. 